\title{
Presence of Tertiary Lymphoid Organ in Nasal Inverted Papilloma Is Correlated with Eosinophil Infiltration and Local Immunoglobulin Production
}

\author{
Qing Bao ${ }^{a}$ Xue-Xue Guo ${ }^{a}$ Chen Cao ${ }^{a}$ Qian-Ying Li ${ }^{a}$ Lin Sun ${ }^{a}$ Xiao-Yan Ye \\ Li-Yue Lia Jun-Chao Dong $^{b}$ Yi-Fang Gaoc He-Xin Chen ${ }^{\mathrm{a}}$ Chun-Wei Lia \\ ${ }^{a}$ Department of Otolaryngology, Guangzhou Key Laboratory of Otorhinolaryngology, The First Affiliated Hospital, \\ Sun Yat-sen University, Guangzhou, China; 'bepartment of Immunology, Zhongshan School of Medicine, \\ Sun Yat-sen University, Guangzhou, China; ' Organ Transplantation Centre, Guangdong Provincial Key Laboratory of \\ Organ Donation and Transplant Immunology, The First Affiliated Hospital, Sun Yat-sen University, \\ Guangzhou, China
}

\section{Keywords}

Nasal inverted papilloma - Tertiary lymphoid organs . Local immunoglobulins · Eosinophils · Th2 responses

\begin{abstract}
Introduction: Nasal inverted papilloma (NIP) is a benign tumour with multiple inflammatory cell infiltration. Tertiary lymphoid organs (TLOs) support local antibody production and play important roles in airway inflammation. However, the evidence of TLOs and local immunoglobulins in NIP has not been reported yet. We investigated the presence of TLOs and immunoglobulins in NIP tissues and their association with the clinical-pathological characteristics of NIPs. Methods: We analyzed the occurrence and composition of TLOs and local immunoglobulins by immunohistochemistry and evaluated the lymph organogenesis associated genes and cytokines by quantitative qPCR and Luminex assays, respectively, in papilloma tissues from 84 NIP cases. Results: TLOs were present in 54\% (45/84) of the NIP patients but not in control subjects. TLOs were composed of T cells, B cells, fol-
\end{abstract}

licular dendritic cells, macrophages, and natural killer cells. Compared to NIP tissues without TLOs, tissues with TLOs showed significantly higher eosinophil infiltration levels (3.5-fold), elevation of lymphorganogenic genes (CXCL12, CXCL13, CCL20, CCL21, CD21L, and lymphotoxin alpha and beta), and increased Th17 (IL-21, IL-22, and GM-CSF) and Th2 (IL-5 and IL-13) cytokine production. Moreover, NIP with TLOs demonstrated a higher number of follicular T helper cells and immunoglobulin-producing plasma cells (CD138+ $\lg \mathrm{A}+, \mathrm{CD} 138+\lg \mathrm{M}+, \mathrm{CD} 138+\lg \mathrm{E}+$, and CD138+ $\lg \mathrm{G}+$ ) than those without TLOs, and these antibody-producing cells were positively correlated with the eosinophil number. Conclusion: The high frequency of TLOs and excess local immunoglobulin production are associated with an eosinophilic and Th2 skew microenvironment in the NIP mucosa, which would contribute to an important immunopathogenic response during NIP pathogenesis.

c) 2020 S. Karger AG, Basel

Qing Bao and Xue-Xue Guo contributed equally to this paper. Edited by: H.-U. Simon, Bern.
Chun-Wei Li or He-Xin Chen 


\section{Introduction}

A nasal inverted papilloma (NIP) is a type of benign nasal tumour with a significant propensity for recurrence. Grossly, NIPs appear as enlarged mucosal polypoid lesions and often need to be differentially diagnosed from nasal polyps by histopathological evaluation. We previously demonstrated an increase in NIP tissue infiltration by multiple inflammatory cells in Chinese patients, with a neutrophil-dominant phenotype, and that the local increase in IL-17 is associated with neutrophilia in NIPs [1, 2]. An earlier study performed in Caucasian patients with nasal papilloma also reported that inflammatory cell numbers were greater in NIP than in other types of papilloma, such as cylindrical papilloma and exophytic squamous papilloma $[3,4]$. The above evidence suggests that local inflammation as well as immune activation may be enhanced in the NIP tissue environment and may be involved in the pathogenesis of NIP.

Tertiary lymphoid organs (TLOs; tertiary lymphoid structures or ectopic lymphoid tissues) are found in local tissues affected by acute or chronic inflammation as a result of infection, autoimmunity, cancer, and allograft rejection [5]. Similar to conventional lymphoid organs, TLOs have segregated B-cell and T-cell areas, follicular cell networks, a stromal reticulum, and endothelial venules, as well as other infiltrating immune cells (such as dendritic cells [DCs], macrophages, and natural killer cells). Therefore, TLOs are considered to have activity similar to that of germinal centres and contribute to the local immune response. The roles of TLOs in various diseases are different, and both beneficial and deleterious outcomes have been reported. Bronchus TLOs may play protective roles in the context of acute respiratory viral or bacterial infections [6]. However, in chronic or recurrent inflammatory conditions (such as COPD, asthma, and chronic rhinosinusitis [CRS]), TLOs are essential for local B-cell activation and antibody production, which in turn activate macrophages, eosinophils, or neutrophils, leading to tissue damage [6-8]. Most studies have demonstrated that in cancer, tumour-associated TLOs are associated with a favourable prognosis and may present anti-tumour responses [9].

The high level of infiltration by neutrophils as well as lymphocytes, eosinophils, and macrophages and the increase in IL-17 production in NIP tissues may suggest that there is an active immune response to pathogens in the local mucosa [2]. TLOs are present when there is a continual need for leucocyte effusion in response to persistent antigens at the site of microbial infection. How-

Tertiary Lymphoid Organ in Inverted Papilloma ever, to date, the occurrence of TLOs as well as local antibodies in benign nasal tumours such as NIPs has not been demonstrated. The purpose of the current study was to determine whether TLOs are present in NIP tissues and their association with the clinical and pathological characteristics of NIP. Moreover, the relationship between TLOs and local immunoglobulin production was also investigated. By using histology/immunohistochemistry and immunofluorescent evaluation and the determination of signature cytokines in papilloma tissues from patients with NIP, we found that TLOs were present in $54 \%$ of the NIP patients and the appearance of TLOs was associated with a higher level of tissue eosinophil infiltration and elevated local Th2 and Th17 microenvironment; in addition, the levels of local immunoglobulins (IgA, IgM, IgE, and IgG) were higher in NIP tissues with TLOs than in those without TLOs. Collectively, for the first time, our results suggest that the prevalence of TLOs in NIP is relatively high and is associated with the eosinophilic phenotype of NIP. The presence of TLOs may support local immunoglobulin production in NIP tissues.

\section{Materials and Methods}

Subjects and Samples

Patients with NIPs $(N=84)$ were recruited from the Department of Otolaryngology, the First Affiliated Hospital of Sun Yatsen University, Guangzhou, China. The diagnosis of NIP was confirmed by ENT physicians and pathologists based on the symptoms, computerized tomography scans, endoscopic examinations, and histological evaluation of tissue biopsies. NIP tissues were obtained from patients who underwent functional endoscopic sinus surgery. The demographic and clinical information, including age, gender, smoking habit, Krouse staging system, the concomitant presence of nasal polyps or allergic rhinitis, and history of NIP surgical operations, is shown in online suppl. Table E1; for all online suppl. material, see www.karger.com/doi/10.1159/000510966. Healthy nasal mucosa was obtained from control subjects $(n=18)$ with nasal septal deviation or a nasal fracture who underwent septoplasty. The control subjects were confirmed to have no signs or symptoms of NIP, CRS, or allergic rhinitis. Tissue samples were processed by formalin fixation and paraffin embedding (FFPE), tissue homogenization, and storage in RNA stabilizers.

\section{Evaluation of Immune Cells and Immunoglobulins in NIP}

Tissues

FFPE tissues were sliced into $10-\mu \mathrm{m}$ thick sections for histological, immunohistochemical, and immunofluorescent staining experiments. All tissues were stained with haematoxylin and eosin $(\mathrm{H} \& \mathrm{E})$ to assess the general histological evaluation, including the TLO formation and degree of eosinophil infiltration.

Immune cells were determined by using immunohistochemistry of candidate markers, including neutrophil elastase, CD3, CD4, CD8, CD20, CD138, CD21, CD68, and CD56 (details of antibody 


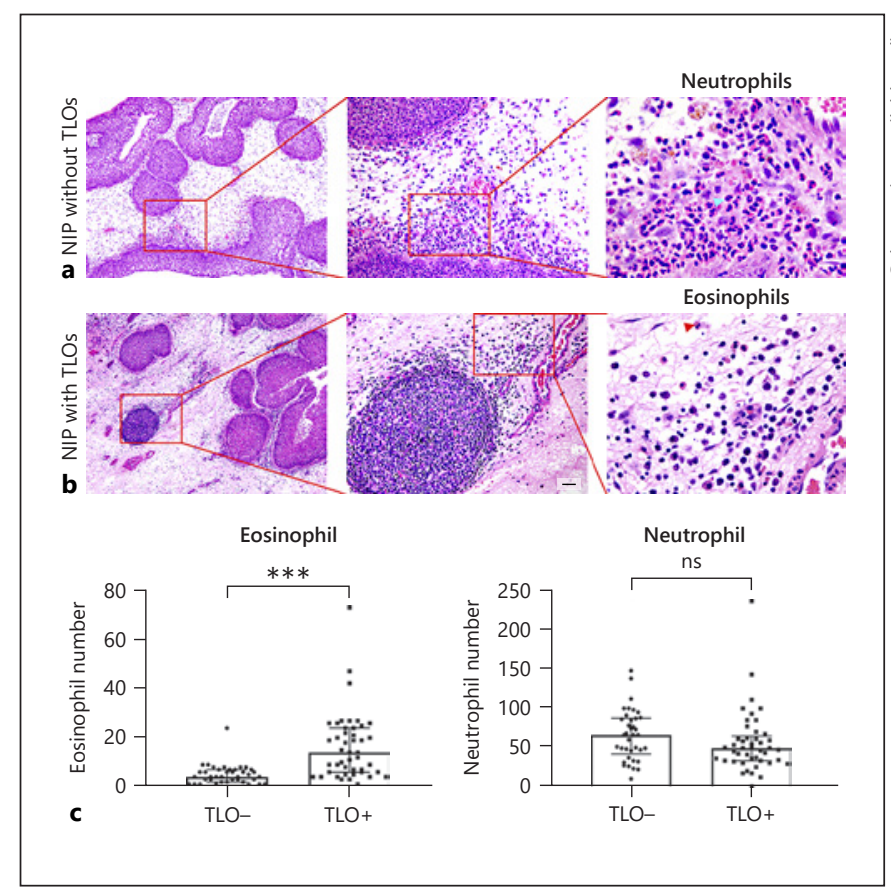

Fig. 1. Representative pictures of TLOs by H\&E staining in patients with NIP. Neutrophilia was observed in most NIP tissues (a), while NIP with TLOs was associated with eosinophilia (b). c The numbers of eosinophils and neutrophils were compared in the tissues with TLOs $(n=45)$ versus without TLOs $(n=39)$. Original magnification is $\times 100$ and $\times 200$ (scale bar $=50 \mu \mathrm{m})$, respectively. Scatter plot with bar is shown with the median values. ${ }^{* * *} p<0.001$. TLOs, tertiary lymphoid organs; NIP, nasal inverted papilloma.

information are listed in online suppl. Table E2). Immunoglobulin-producing B cells and follicular helper $\mathrm{T}(\mathrm{Tfh})$ cells were determined by using immunofluorescent staining of candidate markers, including IgA, IgM, IgE, IgG, CD138, CXCR5, and CD4 (online suppl. Table E2). Details of immunohistochemical and immunofluorescent experiments are described in the online suppl. material.

\section{Determination of mRNA Levels of TLO-Related Genes}

mRNA levels of TLO-related genes (CXCL12, CXCL13, CCL19, CCL20, CD21L, LTa, and LT $\beta$ ) were determined by quantitative RT-PCR. The relative mRNA expression levels were calculated by the $2^{-\Delta C t}$ method which was normalized by the housekeeping gene GAPDH. The primer sequences for the genes are shown in online suppl. Table E3. Details of RNA extraction and quantitative RTPCR assay are described in the online suppl. material.

Cytokine Levels in Tissue Samples

Cytokine levels of IL-1 $\beta$, IL-2, IL-6, TNF- $\alpha$, IL-12p70, IL-18, IFN- $\gamma$, IL-4, IL-5, IL-9, IL-13, IL-17, IL-21, IL-22, IL-23, and GMCSF in tissue homogenates were measured by customized ProcartaPlex assay (Thermo Fisher Scientific) following the manufacturer's instructions. Details of tissue homogenates and Luminex assay were described in the online suppl. material.
Table 1. Correlation of TLOs with clinicopathological characteristics

\begin{tabular}{|c|c|c|c|}
\hline \multirow[t]{2}{*}{ Characteristics } & \multicolumn{2}{|l|}{ TLO } & \multirow[t]{2}{*}{$p$ value } \\
\hline & absence & presence & \\
\hline \multicolumn{4}{|l|}{ Age, years } \\
\hline$<50$ & $15(37 \%)$ & $25(63 \%)$ & 0.131 \\
\hline$\geq 50$ & $24(55 \%)$ & $20(45 \%)$ & \\
\hline \multicolumn{4}{|l|}{ Gender } \\
\hline Male & $31(45 \%)$ & $38(55 \%)$ & 0.581 \\
\hline Female & $8(53 \%)$ & $7(47 \%)$ & \\
\hline \multicolumn{4}{|l|}{ Smoking } \\
\hline Smoker & $17(57 \%)$ & $13(43 \%)$ & 0.178 \\
\hline Non-smoker & $22(41 \%)$ & $32(59 \%)$ & \\
\hline \multicolumn{4}{|l|}{$\mathrm{NP}$} \\
\hline With NP & $10(36 \%)$ & $18(64 \%)$ & 0.254 \\
\hline Without NP & $29(52 \%)$ & $27(48 \%)$ & \\
\hline \multicolumn{4}{|l|}{ Recurrence } \\
\hline First diagnosis of NIP & $24(53 \%)$ & $21(47 \%)$ & 0.195 \\
\hline Recurrent NIP & $15(38 \%)$ & $24(62 \%)$ & \\
\hline \multicolumn{4}{|l|}{ Krouse staging system } \\
\hline Stage I and II & $10(42 \%)$ & $14(58 \%)$ & 0.634 \\
\hline Stage III & $29(48 \%)$ & $31(52 \%)$ & \\
\hline \multicolumn{4}{|l|}{ Eosinophils in tissues* } \\
\hline Eosinophilia & $1(3 \%)$ & $28(97 \%)$ & $<0.001$ \\
\hline Non-eosinophilia & $38(69 \%)$ & $17(31 \%)$ & \\
\hline
\end{tabular}

TLOs, tertiary lymphoid organs; NIP, nasal inverted papilloma; $\mathrm{NP}$, nasal polyp. * Eosinophilia is defined by the eosinophil number $\geq 10$ in NIP, tissue sections which are observed under $\times 400$ magnification.

\section{Statistical Analysis}

The Mann-Whitney U test was used to compare the infiltration by eosinophils and neutrophils, mRNA levels of selected genes, and cytokine production levels between 2 independent groups. The Kruskal-Wallis test followed by Dunn's multiple comparisons test was used to compare the inter-group differences in $>3$ groups. The $\chi^{2}$ test was applied to compare the differences in proportions between the TLO-positive and TLO-negative groups and clinical parameters. The Spearman rank test was used to analyze the relationship between the infiltration by eosinophils or neutrophils and immunoglobulin levels. All statistical analyses were performed, and graphs were produced using GraphPad Prism version 8.0 (GraphPad Software, San Diego, CA, USA).

\section{Results}

\section{Increased Frequency of TLO Formation in NIPs Is} Associated with Eosinophilia

Papilloma tissues from a cohort of patients with NIP $(N=84)$ were used to determine the occurrence and prevalence of TLOs by histological examination. A TLO-like 


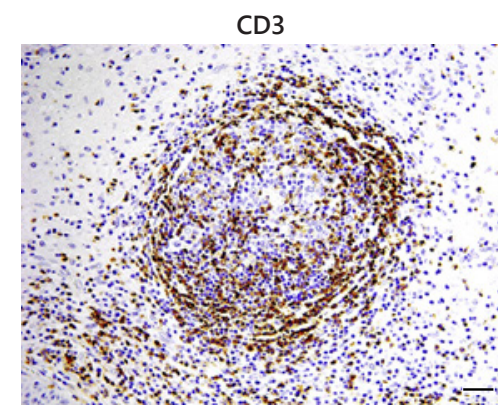

CD20

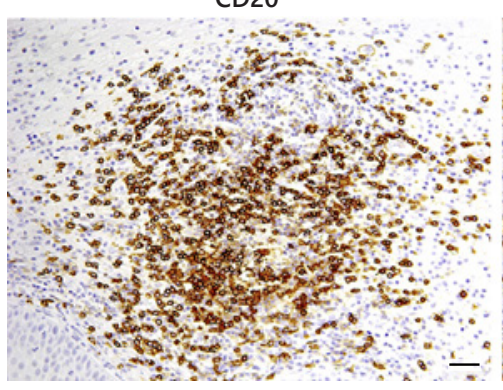

CD68

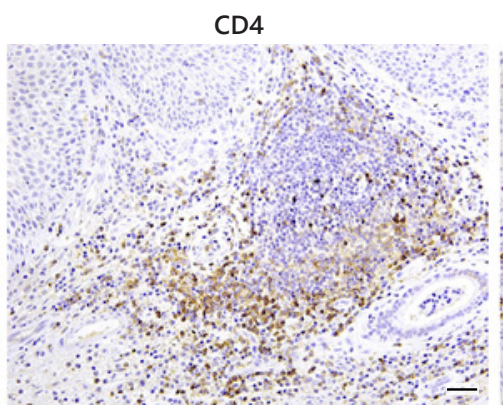

CD138

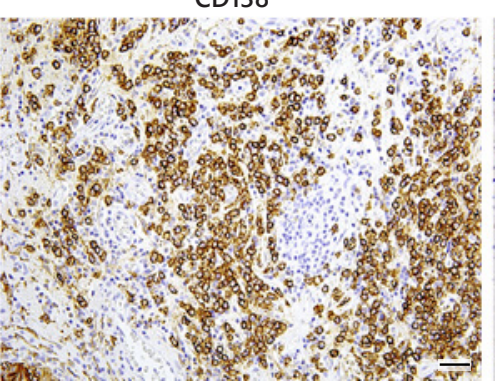

CD56

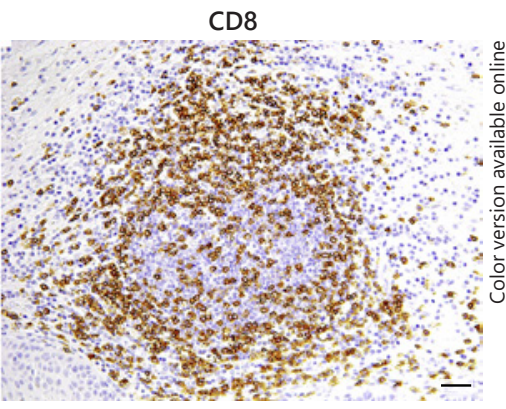

CD21
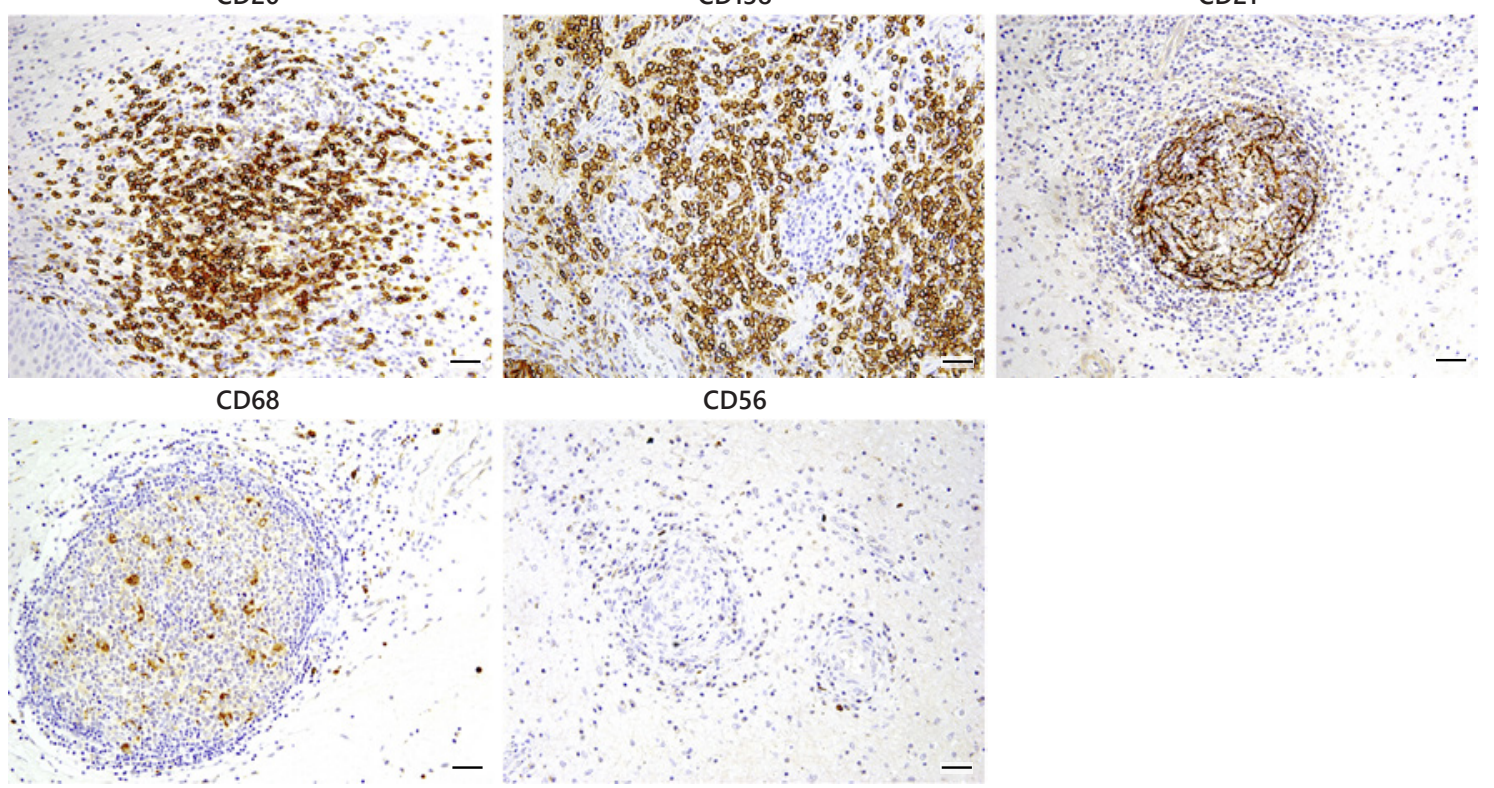

Fig. 2. Representative pictures of the immune cells infiltrating in TLOs, including CD3+ T cells, CD4+ T cells, CD8+ T cells, CD20+ B cells, CD138+ plasma B cells, CD21+ follicular DCs, CD68+ macrophages, and CD56+ nature killer cells. Original magnification is $\times 200$ (scale bar $=50 \mu \mathrm{m}$ ). TLOs, tertiary lymphoid organs; DCs, dendritic cells.

structure was defined as organized lymphoid aggregates occurring in the lamina propria of the mucosa (Fig. 1). These TLO-like structures were observed in 54\% of NIP patients ( 45 out of 84 cases) but not in control subjects. To confirm that these germinal centre (GC)-like structures were indeed TLOs, the cellular formations inside the lymphoid aggregates were characterized in tissues from representative patients. The TLO-like structures were mainly composed of T cells (by the determination of CD3+, CD4+, and CD8+ cells), B cells (by the determination of CD20+ and CD138+ cells), and follicular DCs (by the determination of CD21+ cells) (Fig. 2); moreover, innate immune cells such as macrophages (by the determination of CD68+ cells) and natural killer cells (by the determination of CD56+ cells) were found inside or surrounding the follicle structures (Fig. 2). Hence, a variety of immune cells indicate an active GC network, confirming the presence of TLOs in mucosal tissues from NIP patients.

Next, we analyzed the relationship between the appearance of TLOs and the clinical and pathological characteristics within the NIP patient group. TLO occurrence was significantly associated with tissue eosinophilia (Table 1), while there was no significant relationship between the presence of TLOs and age, gender, smoking, concurrent nasal polyps, previous surgery, or tumour grade (Krouse stage) (Table 1). In addition, tissues with TLOs showed significantly higher eosinophil infiltration levels (3.5-fold) than those without TLOs, but the numbers of neutrophils were similar between TLO+ and TLO- tissues (Fig. 1). 


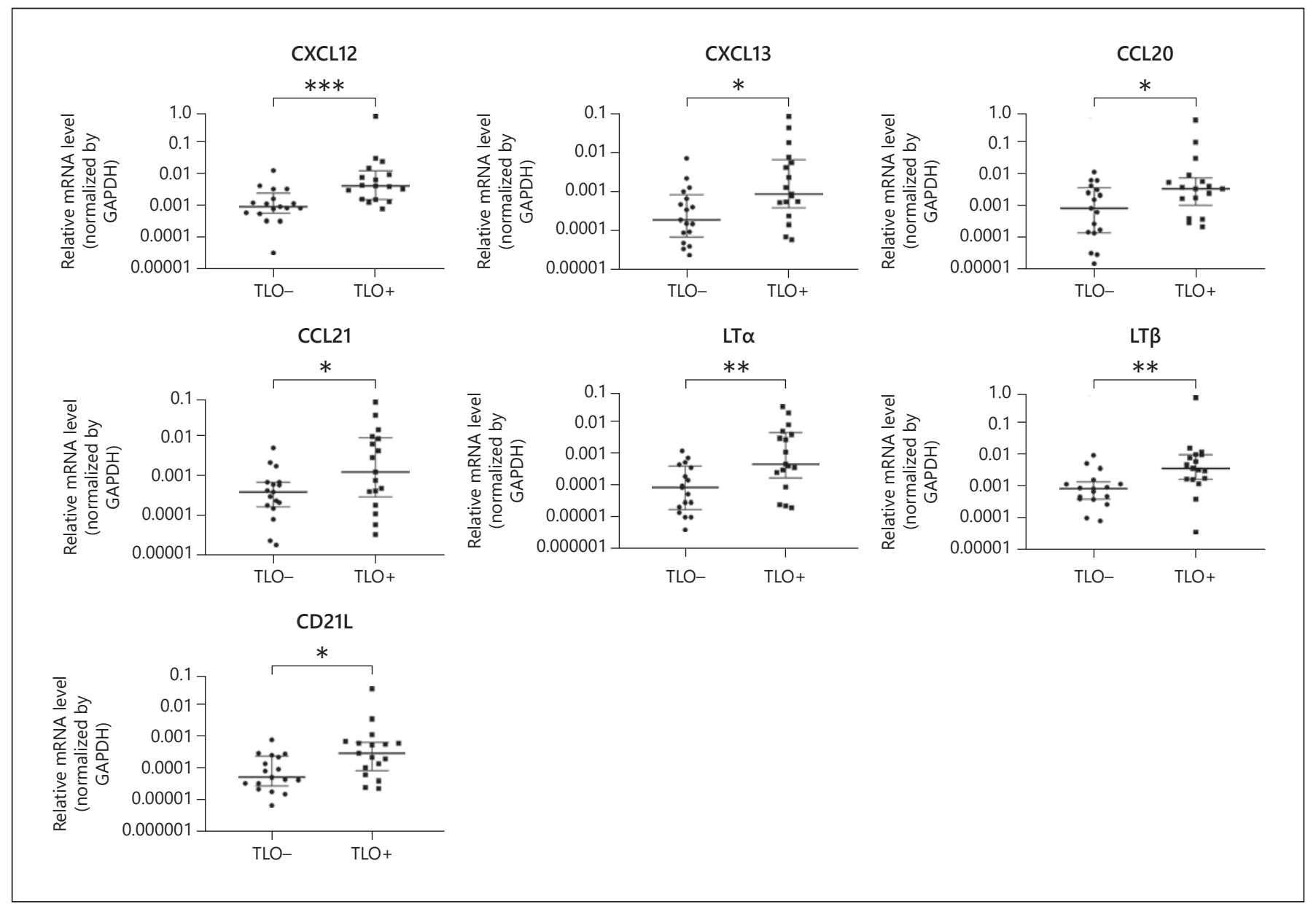

Fig. 3. mRNA expression levels of lymphorganogenic genes in NIP tissues with TLOs $(n=17)$ and without TLOs $(n=17)$, including CXCL12, CXCL13, CCL20, CCL21, LTa, LT $\beta$, and CD21L. Scatter plot is shown with the median values. ${ }^{*} p<0.05,{ }^{*} p<0.01$, and ${ }^{* * *} p<0.001$. TLOs, tertiary lymphoid organs; NIP, nasal inverted papilloma.

Upregulation of the Expression of Chemokine Genes Associated with Lymphoid Organization in NIPs with TLOs

The chemokine genes (including CXCL12, CXCL13, CCL20, CCL21, CD21L, and lymphotoxin $\beta$ receptor $[\mathrm{LT} \beta \mathrm{R}]$ ligands [LTa and LT $\beta]$ ) relevant to TLO formation and organization were examined in NIPs with TLOs versus those without TLOs as well as in the control mucosa. The mRNA levels of the aforementioned chemokine genes were all higher in tissues with TLOs than in those without TLOs in NIP patients (CXCL12, 4.5-fold; CXCL13, 4.7-fold; CCL20, 3.9-fold; CCL21, 3.1-fold; LTa, 4.8-fold; LT $\beta$, 4.4-fold) (Fig. 3). The increase in the levels of these lymphorganogenic factors could be an important upstream mechanism involved in the TLO initiation and development in papilloma tissues.
Enhanced Th17 and Th2 Responses in the Microenvironment of Tissues with TLOs

Panels of cytokines (including IL-1 $\beta$, IL-2, IL-6, TNF- $\alpha$, IL-12p70, IL-18, IFN- $\gamma$, IL-4, IL-5, IL-9, IL-13, IL-17, IL-21, IL-22, IL-23, and GM-CSF) were analyzed in the tissue homogenate to evaluate the immune characteristics of the tissue microenvironment associated with TLOs. The results showed that significant increases in the levels of Th17 (IL-21, 1.7-fold; IL-22, 2.0-fold; GM-CSF, 1.5-fold) and Th2 (IL-5, 1.6-fold; IL-13, 1.9-fold) response cytokines and other pro-inflammatory cytokines (IL-6, 2.2-fold; TNF- $\alpha$, 1.5-fold) were present in NIPs with TLOs versus those without TLOs (Fig. 4). The eosinophilic infiltration in NIPs was associated with TLO presence; consequently, we found increased levels of the Th2-type cytokines IL-5 and IL-13 in tissues with TLOs. 


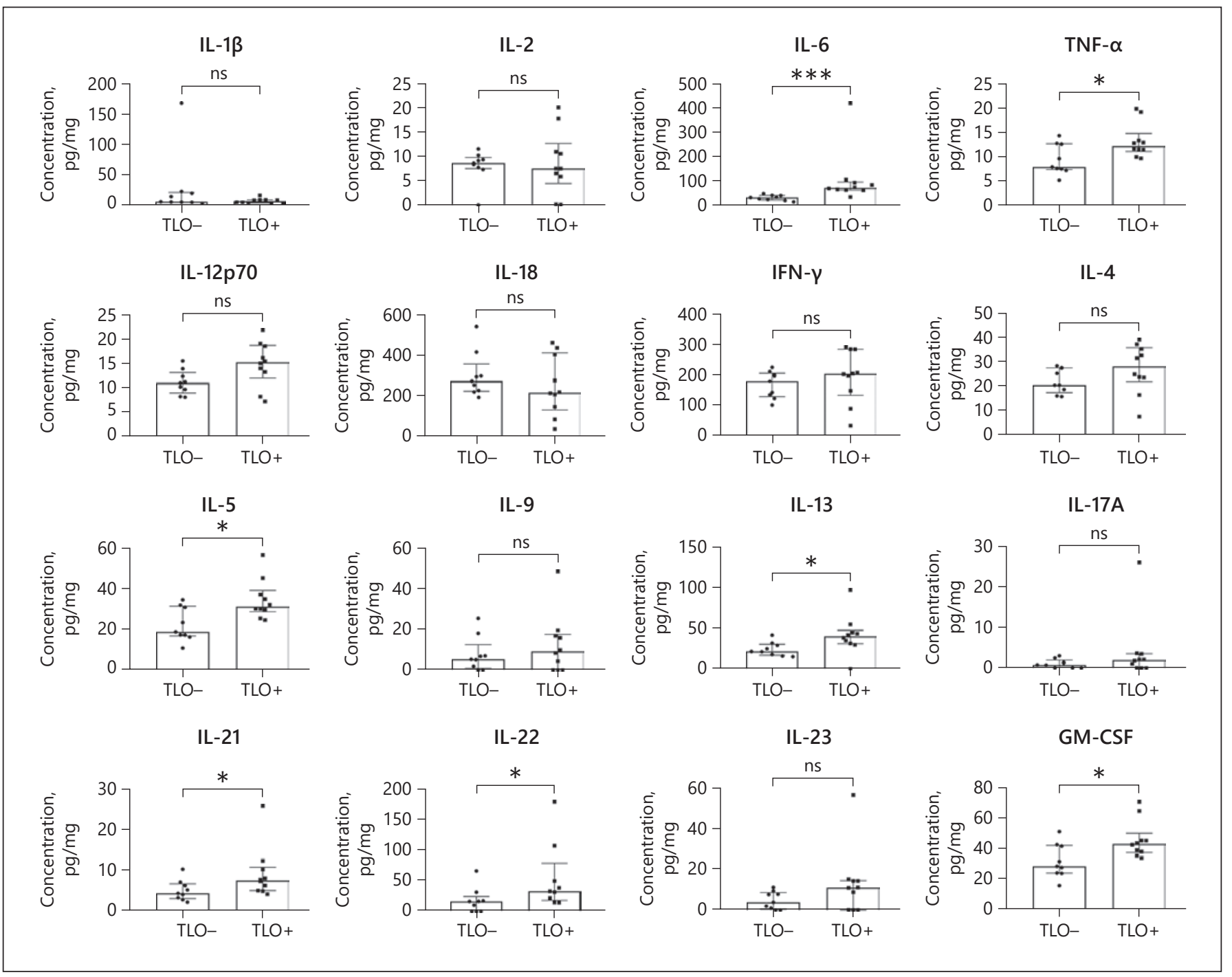

Fig. 4. Concentration of cytokines in NIP tissues with TLOs $(n=10)$ and without TLOs $(n=9)$, including IL-1 $\beta$, IL-2, IL-6, TNF- $\alpha$, IL-12p70, IL-18, IFN- $\gamma$, IL-4, IL-5, IL-9, IL-13, IL-17, IL-21, IL-22, IL-23, and GM-CSF. Scatter plot with bar is shown with the median values. ${ }^{*} p<0.05,{ }^{*} p<0.01$, and ${ }^{* * *} p<0.001$. TLOs, tertiary lymphoid organs; NIP, nasal inverted papilloma.

The above results show that the increase in Th17- and Th2-type cytokines in the microenvironment may favour TLO development in NIP tissues.

\section{Increase in the CXCR5+ Follicular T Helper Cell \\ Proportion in NIPs with TLOs}

CXCR5+ Tfh cells are required for the formation and maintenance of B-cell follicles in both secondary lymphoid organs and TLOs $[10,11]$. CXCR5+ Tfh cells are recruited by their specific ligand (CXCL13) and other cytokines, such as IL-6, the expression of which was also upregulated in NIP tissues with TLOs in the above results.
Consistent with previous reports showing the appearance of CXCR5+ CD4+ Tfh cells in TLOs in the airway mucosa, we found greater Tfh cell infiltration in NIPs with TLOs than in those without TLOs and in controls (Fig. 5). These results indicate that Tfh cells inside NIP-tissue TLOs may be responsible for promoting B-cell activation and antibody generation.

\section{Increase in Local Immunoglobulin Production in}

Tissues with TLOs

The mucosal-associated lymphoid tissues in the respiratory tract, gut, and colon are considered important 


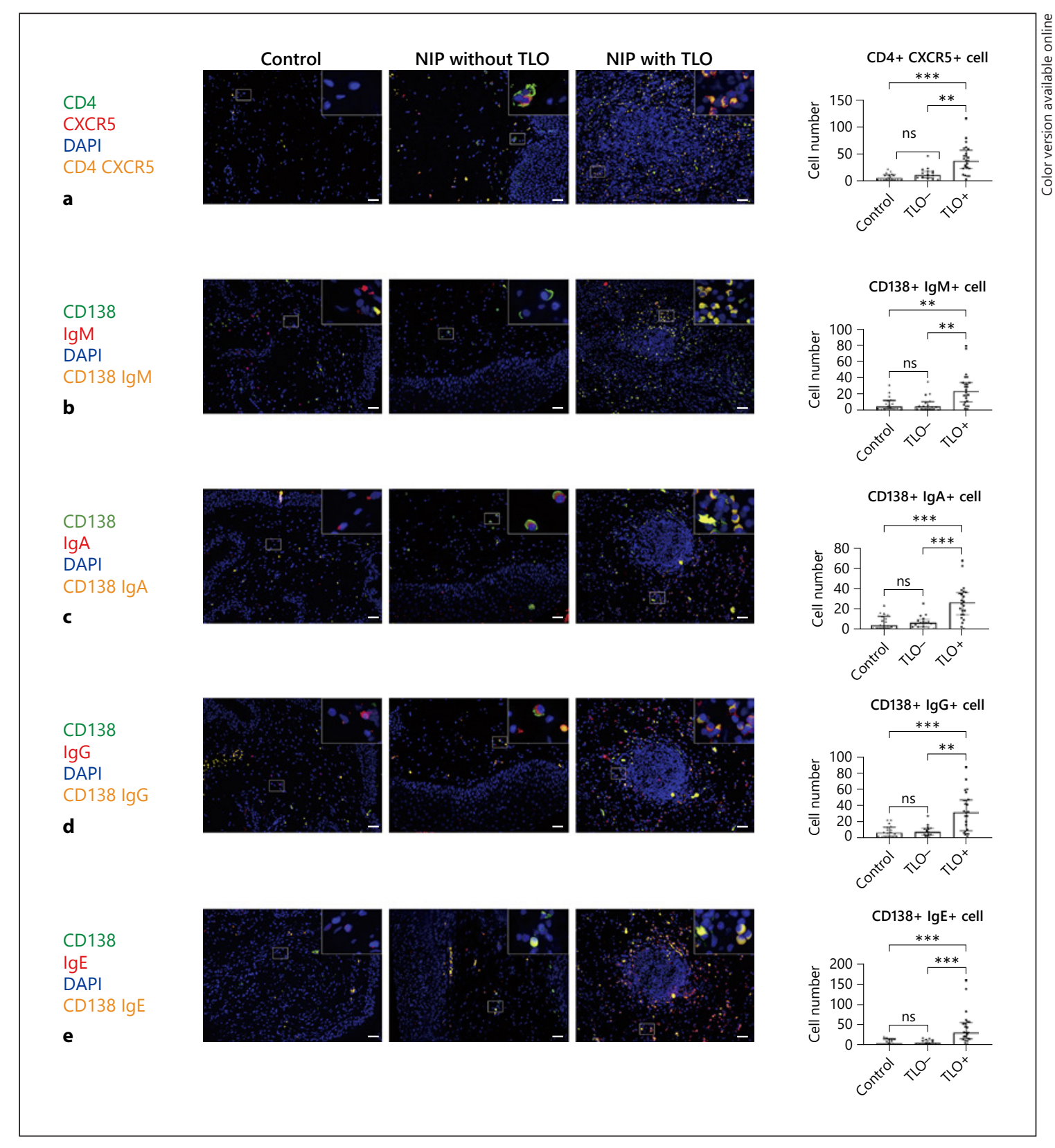

Fig. 5. Tfh cells and local immunoglobulin-producing plasma $\mathrm{B}$ cells in control nasal mucosa $(n=18)$, NIP without TLOs $(n=16)$, and NIP with TLOs $(n=24)$. Representative immunofluorescent images show staining for CXCR5+ CD4+ Tfh cells (a), CD138+ IgM+ plasma B cells (b), CD138+ IgA+ plasma B cells (c), CD138+ IgG+ plasma B cells (d), and CD138+ IgE+ plasma B cells (e). The

"sources" of local antibody production [7, 9]. B cells in TLOs can be activated directly at sites of mucosal inflammation and generate various immunoglobulins. We then explored local immunoglobulin production in NIPs and its association with TLOs. The levels of different types of numbers of Tfh cells, CD138+ IgM+ cells, CD138+ IgA+ cells, CD138+ IgG+ cells, and CD138+ IgE+ cells were compared in control, NIP without TLOs, and NIP with TLOs. Scatter plot with bar is shown with the median values. ${ }^{*} p<0.05,{ }^{*} p<0.01$, and ${ }^{* * *} p<$ 0.001 . Tfh cells, follicular helper T cells; TLOs, tertiary lymphoid organs; NIP, nasal inverted papilloma. 
most of the tissue samples, whereas a significant increase in $\operatorname{Ig} \mathrm{A}+, \operatorname{IgM}+, \operatorname{IgE}+$, and $\operatorname{IgG}+$ plasma cells was found in NIP tissues with TLOs (Fig. 5). These antibody-producing plasma cells were mainly localized around the border of TLOs and distributed in extrafollicular regions (Fig. 5). These results confirm that immunoglobulins can be produced locally by plasma cells and TLOs are critical for local antibody production in NIP tissues.

We also examined the association between immunoglobulin levels and the clinical-pathological characteristics of NIP. The amounts of IgA+, IgM+, IgE+, and IgG+ plasma cells were positively correlated with eosinophil infiltration and the Tfh cell number, but no correlation with neutrophils was observed (online suppl. Fig. E1). We did not find a difference in immunoglobulin-producing cells associated with the following clinical parameters: age, sex, history of recurrence, concomitant presence of nasal polyps, smoking habit, and Krouse stage (data not shown here).

\section{Discussion}

Two recent studies have reported evidence of TLOs in CRS [7, 8]. Song et al. [7] found that TLOs are present in $20 \%$ of tissues from patients with CRS with nasal polyps (CRSwNP) but almost absent in patients with CRS without nasal polyps, whereas Lau et al. [8] found that $37 \%$ of tissues from patients with recalcitrant CRSwNP have TLOs. NIPs present as enlarged mucosal polypoid lesions and have a gross appearance similar to that of nasal polyps. As NIP is a type of benign tumour, the pathological changes in the mucosa are more severe than those of inflammatory polyps. For the first time, we report that TLOs exist in NIP tissues and the frequency of TLOs (54\%) in NIP samples seems to be higher than that in polyps. We also found that TLO formation is significantly associated with eosinophilic NIP; moreover, we found increased local immunoglobulin-producing plasma B cells in NIP tissues with TLOs.

TLOs develop after repeated exposure to microbes, pathogens, or inflammatory stimuli $[12,13]$. The current results demonstrated an increase in tissue inflammatory microenvironment which could potentially contribute to the TLO development. First, the recruitment of granulocytes (neutrophils or eosinophils) seems to be a prominent feature in tissues from different organs with TLOs $[6,14,15]$. Second, upregulation of lymphorganogenic chemokines was found in NIP tissues with TLOs. CXCL12, CXCL13, CCL20, and CCL21 are specific ligands for

Tertiary Lymphoid Organ in Inverted Papilloma
CXCR4, CXCR5, CCR6, and CCR7, respectively, and they are regarded as homeostatic chemokines that recruit lymphocytes, direct their homing to the appropriate area, and promote T-cell and B-cell survival $[16,17]$. LTa and LT $\beta$ are secreted by local immune cells, and then TLO stromal cells (mainly follicular DCs and endothelial cells) are stimulated to initiate the recruitment of haematopoietic cells [17]; CD21L is responsible for activating CD21+ follicular DCs [17]. Finally, increase of various types (especially Th17 and Th2) of pro-inflammatory cytokines in NIPs with TLOs is observed. The upregulation of Th17type cytokines, including IL-21, IL-22, and GM-CSF, is important for promoting TLO development in response to pathogens; for example, these cytokines induce stromal cell production of homeostatic chemokines, leading to lymphorganogenesis $[16,18,19]$; IL-21 also plays a central role in Tfh cell differentiation, which in turn differentiates B cells into plasma cells and generates highaffinity antibodies [20]. We previously reported that papilloma tissues exhibit high levels of infiltration by multiple inflammatory cells with neutrophil dominance and the IL-17 response is enhanced in NIP tissues $[1,2]$. The overexpression of Th2-type cytokines (IL-5 and IL-13) has been shown to contribute to the induction of bronchus- or gut-associated lymphoid tissues [21, 22]. Moreover, increased levels of other pro-inflammatory cytokines (IL- 6 and TNF- $\alpha$ ) in NIPs with TLOs have also been reported to contribute to TLO organization and maintenance, for example, the recruitment and survival of multiple types of immune cells [23-25]. Taken together, the above findings suggest that the local inflammatory milieu and homeostasis mechanisms operate simultaneously to promote continuous TLO expansion in NIPs.

The appearance of TLOs seems to be beneficial in the context of acute airway infection, in which TLOs are responsible for producing specific antibodies to accelerate the clearance of pathogens $[9,12]$. However, in chronic inflammatory airway mucosa, TLOs are often associated with the severity of diseases, such as COPD, asthma, and recurrent CRSwNP [6-8]. Hence, TLOs are a doubleedged "sword" during chronic inflammation, that is, the formation of TLOs is a consequence of the host innate immune response to limit pathogen infection, but at the same time, it may lead to greater and faster immune responses in the local mucosa, which exacerbate chronic disease. NIP is generally considered a kind of benign tumour, but the high level of inflammatory cell infiltration suggests that certain inflammatory mechanisms likely play important roles in NIP pathogenesis. We found that in NIP tissues, the increase in TLO occurrence was accompanied by the

Int Arch Allergy Immunol 2021;182:350-359 357 
upregulation of immunoglobulin production, and this finding correlated with the high level of infiltration by eosinophils. We hypothesize that the local antibodies produced by TLOs such as IgA, IgE, and IgG can potently induce eosinophil and mast cell degranulation, leading to the secretion of reactive oxygen species and other inflammatory mediators. Therefore, the presence of TLOs and enhanced local immunoglobulin production may be associated with eosinophil-mediated tissue damage in NIP, but the causal relationship between TLOs, local antibody production, and eosinophilia in NIP remains unclear.

This study found that the hyperproduction of local immunoglobulins was prominent in NIP tissue with TLOs, but fewer antibodies (especially IgE) were observed in tissues without TLOs. These results indicate that distinct pathogenic stimuli may drive antigen-specific B-cell expansion. The pathological roles of local IgE in eosinophilic airway inflammation (such as CRSwNP and asth$\mathrm{ma}$ ) have been revealed in several studies [26]. The presence of local IgE antibodies, for example, IgE specific for enterotoxins from Staphylococcus aureus, can induce histamine release in the polyp tissue and may serve as a biomarker of the severity of the diseases [27, 28]; furthermore, in an experimental model, mice challenged with OVA had an increased number of OVA-specific IgE-secreting plasma cells in TLOs in the lungs, which was associated with airway hyperresponsiveness [29]. Sustained exposure to pathogens, such as human papilloma virus, in the NIP mucosa is hypothesized to be involved in the pathogenesis of NIP [30]. Our results imply that certain dominant B-cell clones in TLOs may be generated in response to specific pathogenic antigens in NIP. Analyzing the epitope patterns in these local immunoglobulins and "fishing" out the corresponding antigens deserve further investigation and would be helpful to discover the triggers during NIP pathogenesis.

An increase in neutrophil infiltration occurred in almost all samples in the current study and in our previous studies performed in Chinese NIP patients [1, 2]. It seems that neutrophilia is a very common inflammatory phenotype in NIP tissues from Chinese patients. We found a certain portion of samples with eosinophilia that was closely associated with TLO occurrence, local antibody production, and Th2 and Th17 responses; this pattern may represent a certain pathogenic mechanism in NIP. In chronic airway inflammation (such as asthma and CRS), based on the relative numbers of eosinophils and neutrophils, 4 inflammatory subtypes are commonly described, including eosinophilic, neutrophilic, mixed granulocytic, and paucigranulocytic subtypes [31]. Regarding this con- cept, it would be interesting to determine whether eosinophilia plus TLO positivity is a distinct subtype of NIP and its association with clinical or pathological NIP features; this approach would be helpful to understand the pathophysiologic diversity within patients with NIP.

We did not find that TLO formation was significantly associated with any clinical parameters, suggesting that multiple factors impact the clinical presentation of NIP. Previous studies performed in CRSwNP patients have shown that TLO frequency is positively correlated with the number of previous operations $[7,8]$. Indeed, our results demonstrate that patients with prior surgeries had a higher incidence (i.e., recurrent NIP) of TLOs (62\%) than those without prior surgeries (38\%), but the difference did not reach statistical significance. Because the current study is limited by its retrospective design and sample size, a prospective follow-up cohort study is required to investigate whether the appearance of TLOs is a risk factor for the recurrence of NIP. Another limitation of current study is that the secreted (or extracellular) immunoglobulins were not measured in the tissues. Nonetheless, since the local immunoglobulins are mainly produced by plasma B cells, the levels of CD138+ Ig+ cells still could represent the trend of local immunoglobulin levels in nasal mucosa.

In conclusion, we determined that the frequency of TLOs in patients with NIP is high and the excess production of local immunoglobulins is involved in the development of TLOs. The formation of TLOs and increased local antibody levels are associated with eosinophilia and Th2-skewed pathological patterns in NIP, which likely contribute to certain immunopathogenic responses in NIP. Further research is required to study the mechanisms driving TLO formation, the roles of TLOs in NIP development, and the antigen specificity of the local immunoglobulins in TLOs. Such research will contribute to improving our understanding of NIP pathogenesis and facilitate the development of a potential B-cell-based or antibody-based therapeutic strategy for NIP.

\section{Statement of Ethics}

Approval to conduct this study was obtained from the Institutional Review Board of the First Affiliated Hospital of Sun Yat-sen University (Reference No. [2018]264). The study subjects involved in this study provided written informed consent.

\section{Conflict of Interest Statement}

The authors declare that they have no conflicts of interest. 


\section{Funding Sources}

This study was supported by Grants from the National Natural Science Foundation of China (81770983 and 81974139), National Science Foundation of Guangdong Province (2017A030313621), and Guangzhou Science and Technology Programme (201907010038 and 201707010105).

\section{Author Contributions}

C.W.L. and H.X.C. designed the study. Q.B., X.X.G., C.C., Q.Y.L., L.S., X.Y.Y., and L.Y.L. performed sample processing and experiments. Q.B., X.X.G., J.C.D., and Y.F.G. analyzed the results. Q.B. and X.X.G. wrote the manuscript. C.W.L. and H.X.C. revised and edited the manuscript. All authors read and approved the final manuscript.

\section{References}

1 Zhao L, Li CW, Jin P, Ng CL, Lin ZB, Li YY, et al. Histopathological features of sinonasal inverted papillomas in Chinese patients. Laryngoscope. 2016;126(4):E141-7.

2 Cao C, Yu SF, Zhou YT, Guo XX, Guo JB, Wu $\mathrm{CY}$, et al. Increase in IL-17-positive cells in sinonasal inverted papilloma. Clin Otolaryngol. 2020;45(1):47-54.

3 Orlandi RR, Rubin A, Terrell JE, Anzai Y, Bugdaj M, Lanza DC. Sinus inflammation associated with contralateral inverted papilloma. Am J Rhinol. 2002;16(2):91-5.

4 Roh HJ, Procop GW, Batra PS, Citardi MJ, Lanza DC. Inflammation and the pathogenesis of inverted papilloma. Am J Rhinol. 2004; 18(2):65-74.

5 Jones GW, Hill DG, Jones SA. Understanding immune cells in tertiary lymphoid organ development: it is all starting to come together. Front Immunol. 2016;7:401.

6 Hwang JY, Randall TD, Silva-Sanchez A. Inducible bronchus-associated lymphoid tissue: taming inflammation in the lung. Front Immunol. 2016;7:258.

7 Song J, Wang H, Zhang YN, Cao PP, Liao B, Wang ZZ, et al. Ectopic lymphoid tissues support local immunoglobulin production in chronic rhinosinusitis with nasal polyps. J Allergy Clin Immunol. 2018 Mar;141(3):92737.

8 Lau A, Lester S, Moraitis S, Ou J, Psaltis AJ, McColl S, et al. Tertiary lymphoid organs in recalcitrant chronic rhinosinusitis. J Allergy Clin Immunol. 2017;139(4):1371-e6.

9 Teillaud JL, Dieu-Nosjean MC. Tertiary lymphoid structures: an anti-tumor school for adaptive immune cells and an antibody factory to fight cancer? Front Immunol. 2017;8: 830.

10 Muller G, Hopken UE, Lipp M. The impact of CCR7 and CXCR5 on lymphoid organ development and systemic immunity. Immunol Rev. 2003;195:117-35.

11 Couillault C, Germain C, Dubois B, Kaplon $\mathrm{H}$. Identification of tertiary lymphoid structure-associated follicular helper T cells in human tumors and tissues. Methods Mol Biol. 2018;1845:205-22.
12 Randall TD, Mebius RE. The development and function of mucosal lymphoid tissues: a balancing act with micro-organisms. Mucosal Immunol. 2014;7(3):455-66.

13 Pitzalis C, Jones GW, Bombardieri M, Jones SA. Ectopic lymphoid-like structures in infection, cancer and autoimmunity. Nat Rev Immunol. 2014;14(7):447-62.

14 Ravin KA, Loy M. The eosinophil in infection. Clin Rev Allergy Immunol. 2016;50(2):21427.

15 Tecchio C, Micheletti A, Cassatella MA. Neutrophil-derived cytokines: facts beyond expression. Front Immunol. 2014;5:508.

16 Fleige H, Ravens S, Moschovakis GL, Bölter J, Willenzon S, Sutter G, et al. IL-17-induced CXCL12 recruits B cells and induces follicle formation in BALT in the absence of differentiated FDCs. J Exp Med. 2014;211(4):643-51.

17 Buckley CD, Barone F, Nayar S, Bénézech C, Caamaño J. Stromal cells in chronic inflammation and tertiary lymphoid organ formation. Annu Rev Immunol. 2015;33:715-45.

18 Grogan JL, Ouyang W. A role for Th17 cells in the regulation of tertiary lymphoid follicles. Eur J Immunol. 2012;42(9):2255-62.

19 Rangel-Moreno J, Carragher DM, de la Luz Garcia-Hernandez M, Hwang JY, Kusser K, Hartson L, et al. The development of inducible bronchus-associated lymphoid tissue depends on IL-17. Nat Immunol. 2011;12(7): 639-46.

20 Spolski R, Leonard WJ. Interleukin-21: a double-edged sword with therapeutic potential. Nat Rev Drug Discov. 2014;13(5):379-95.

21 Lee JJ, McGarry MP, Farmer SC, Denzler KL, Larson KA, Carrigan PE, et al. Interleukin-5 expression in the lung epithelium of transgenic mice leads to pulmonary changes pathognomonic of asthma. J Exp Med. 1997;185(12): 2143-56.

22 Pearson C, Uhlig HH, Powrie F. Lymphoid microenvironments and innate lymphoid cells in the gut. Trends Immunol. 2012;33(6): 289-96.
23 Goya S, Matsuoka $\mathrm{H}$, Mori M, Morishita $\mathrm{H}$, Kida H, Kobashi Y, et al. Sustained interleukin-6 signalling leads to the development of lymphoid organ-like structures in the lung. J Pathol. 2003;200(1):82-7.

24 Canete JD, Celis R, Moll C, Izquierdo E, Marsal S, Sanmarti R, et al. Clinical significance of synovial lymphoid neogenesis and its reversal after anti-tumour necrosis factor alpha therapy in rheumatoid arthritis. Ann Rheum Dis. 2009;68:751-6.

25 Denton AE, Innocentin S, Carr EJ, Bradford BM, Lafouresse F, Mabbott NA, et al. Type I interferon induces CXCL13 to support ectopic germinal center formation. J Exp Med. 2019;216(3):621-37.

26 Tan BK, Peters AT, Schleimer RP, Hulse KE. Pathogenic and protective roles of $\mathrm{B}$ cells and antibodies in patients with chronic rhinosinusitis. J Allergy Clin Immunol. 2018;141: 1553-60.

27 Chen JB, James LK, Davies AM, Wu YB, Rimmer J, Lund VJ, et al. Antibodies and superantibodies in patients with chronic rhinosinusitis with nasal polyps. J Allergy Clin Immunol. 2017;139(4):1195-e11.

28 Bachert C, Zhang N, Holtappels G, De Lobel L, van Cauwenberge P, Liu S, et al. Presence of IL-5 protein and IgE antibodies to staphylococcal enterotoxins in nasal polyps is associated with comorbid asthma. J Allergy Clin Immunol. 2010;126(5):962-6.

29 Chvatchko Y, Kosco-Vilbois MH, Herren S, Lefort J, Bonnefoy JY. Germinal center formation and local immunoglobulin E (IgE) production in the lung after an airway antigenic challenge. J Exp Med. 1996;184(6): 2353-60.

30 Govindaraj S, Wang H. Does human papilloma virus play a role in sinonasal inverted papilloma? Curr Opin Otolaryngol Head Neck Surg. 2014;22(1):47-51.

31 Taylor SL, Leong LEX, Choo JM, Wesselingh S, Yang IA, Upham JW, et al. Inflammatory phenotypes in patients with severe asthma are associated with distinct airway microbiology. J Allergy Clin Immunol. 2018 Jan;141(1):94103.e15. 